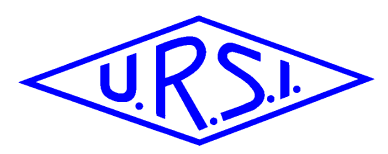

\title{
Again on Spatial Focusing and Shaping of the Electromagnetic Field in the Antenna Radiative Near-Field Region
}

\author{
P. Nepa ${ }^{(1)}$, G. Manara*(1), M. R. Pino ${ }^{(2)}$, R. G. Ayestarán ${ }^{(2)}$ \\ (1) Dept. of Information Engineering, University of Pisa, Pisa, Italy \\ (2) Dept. of Electrical Engineering, University of Oviedo, 33203 Gijón, Spain
}

\begin{abstract}
This paper presents the state-of-the-art on the synthesis techniques and technologies of the antennas for short-range radio links, when specific focusing/shaping of the radiated field in one or more regions close to the antenna itself (inside the antenna radiative near-field region) is required.
\end{abstract}

\section{Introduction}

Near-Field Focused (NFF) antennas are designed to achieve the best radiation performance at one or more focal spots that are located close to the antenna, right outside its reactive near-field region. For electrically large antennas, such a region is referred as the antenna radiative near-field region (or Fresnel region) and it is located between the reactive near-field region and the boundary of the far-field region. NFF antennas are attracting attention since they can be beneficially used in several short-range wireless applications, such as RFID (Radio Frequency Identification) systems, wireless power transfer, microwave imaging, non-destructive sensing and nearfield radiometry, hyperthermia applicators, near-field power combining, characterization of antennas and radar targets in indoor measurement facilities, wireless personal area communications, power/data transfer to bodyimplants, etc.

In 2017, an extended overview of the basic properties, achievable performance and design criteria for NFF antennas has been presented in [1], while a summary of the different technologies used to implement NFF antennas was made available only a few months later in [2]. Since then, a number of research groups presented novel layouts, effective synthesis techniques and valuable applications, for NFF antennas with operating frequencies from microwave frequencies up to tens of $\mathrm{GHz}$.

In this paper, the authors want to give an updated overview with respect the above mentioned review articles [1]-[2], for a better understanding of the actual research trend. Specifically, Sect. 2 discusses the most recent synthesis techniques for NFF antennas, while Sect. 3 refers to some novel layouts. Finally, concluding remarks are drawn in Sect. 4.

\section{Synthesis Techniques for Near-Field Focused Antennas}

In the last few years, novel synthesis techniques have been developed to face with the design of NFF antennas with specific requirements on the figures-of-merit of the focused beam [3]: the size of the focal spot (W) and the side-lobelevel (SLL) at the focal plane, the depth-of-focus (DoF), the focusing gain (FG), the power transfer efficiency (PTE).

Most of the papers dealing with the design of NFF antennas assume that the antenna radiates in free-space. On the other hand, in most application scenarios the presence of the receiving antenna, scatterers at the focal spots or unknown materials close to the antenna may influence the wireless power transfer significantly. Recently, Geyi demonstrated that the technique for the optimization of the PTE between a set of $\mathrm{N}$ transmitting antennas and $\mathrm{M}$ receiving antennas [4] can be efficiently applied in an unknown environment, as long as all the elements of the scattering matrix of the $(\mathrm{N}+\mathrm{M})$-port device have been measured [5]. Here, the PTE is used as the key performance indicator of the radio link, and the proposed approach reduces a field synthesis problem into a circuit analysis problem. Indeed, it is based on the solution of an eigenvalue problem, where the eigenvector related to the maximum eigenvalue represents the excitation vector of the transmitting antennas that maximizes the PTE. The synthesis technique can be applied when the receiving antenna is in the radiative region as well as in the reactive near-field region, given that all the antennas show a negligible return loss, and systematically account for the coupling between the elements of the transmitting and receiving arrays. The effectiveness of the proposed technique has been shown in different application scenarios $[6,7,8]$.

The synthesis of the far-field pattern of an array antenna with specific constraints on its near field (as for example field vanishing close to assigned locations) is not a novel research topic. Nevertheless, the simultaneous near-field and far-field synthesis can appear slightly different in the NFF antenna context, as in this case the main goal is usually the shaping of the near field, with some constraints on the far-field radiation pattern $[9,10]$. As an example, in [10], Ayestarán presented a near-field synthesis technique 
that can account for far-field interference mitigation requirements (namely, an antenna low directivity). In [11], a combination of multitask Bayesian compressive sensing and convex optimization has been used for the near-field pattern synthesis of sparse arrays, with the main goals of reducing the number of radiators with respect to a uniform array and controlling the SLL.

In the context of synthesis techniques suitable for applications where multiple foci may be required, a neuralnetwork based optimization algorithm has been applied to maximize the field amplitude at an assigned set of focal points [12]. It is also worth mentioning the approach introduced in [13], where the phase of the field at assigned focal points can be used as further degrees of freedom to shape the near-field by solving a finite number of convex programming problems. The above method can be used to simultaneously maximize the field at an assigned extended focal region (or multiple focal points) and enforce an upper bound power constraint outside the regions of interest [14]. The above exploitation of the phase shifts has been recently included into a modified multi-target time-reversal approach [15]; although the numerical examples in [15] are relevant to reactive near-field scenarios, the proposed technique can also be applied when the regions of interest are at a larger distance from the array, provided that the assigned focal points are quite close. If the latter is not the case, a simple superposition of the array coefficients synthesized for each distinct focal point may be enough to get a satisfactory performance. The shaping of the (scalar) near-field by resorting to convex optimization has also been applied in [16] when the antenna radiates in freespace, and then extended in [17] to account for the presence of lossy stratified media.

Among the synthesis techniques for aperture antennas, in [18] a NFF reflector antenna has been designed by optimizing the on-axis field through an iterative approach that exploits the Fast Fourier Transform (FFT) to relate the aperture field to the on-axis field. The method was validated by designing a $1.2 \mathrm{~m}$ diameter reflector at $95 \mathrm{GHz}$, with quasi-constant beam radius from $25 \mathrm{~m}$ up to $100 \mathrm{~m}$ from the antenna aperture in the near-field region.

The effect of the amplitude tapering in a planar NFF array has been numerically studied in [19], when all the elements belonging to the same ring of an $8 \times 8$ planar array are fed with a uniform amplitude.

A projection method technique in the spectral (plane wave) domain has been implemented in [20] for the threedimensional shaping of the near-field of an aperture, where the FFT algorithm is used to speed up the synthesis process. The Radial Line Slot Antenna (RLSA) technology at mmwaves (a $12 \lambda$-diameter circular aperture at $30 \mathrm{GHz}$ ) has been used to validate the $3 \mathrm{D}$ synthesis technique.

\section{Novel Layouts and Technolgies}

A novel NFF folded transmitarray for medical applications at $2.45 \mathrm{GHz}$ can be found in [21].The unit-cell of the transmitarray is a square dielectric box with four cylindrical identical holes, where the hole radius is varied to control the phase shift of the transmitted field.

The 20GHz NFF antenna in [22] is made of a cavity-backed holographic metasurface. The antenna surface (a circular disk with a diameter $\mathrm{D}=10 \mathrm{~cm}$ ) is patterned with a sparse array of parallel slots whose position is calculated to focus the radiated field at a distance $\mathrm{F}=10 \mathrm{~cm}$ from the antenna surface. A double-layer structure allows to feed the slotted circular disk from its border, through an annular ring on the periphery of the cavity. The amplitude of the field on the antenna aperture is tapered by controlling the slot size.

Near-field focused arrays in a multi-panel configuration have also been investigated [23], with the main goal of evaluating their effectiveness to shape the near-field $-3 \mathrm{~dB}$ focal spot. The proposed NNF antenna consists of some subarray panels that are arranged around a cylindrical surface, each one being a planar NFF subarray.

As far as NFF reflectarrays are concerned, a large antenna $\left(1.5 \mathrm{~m}^{2}\right.$ wide) has been designed for wireless power transfer at $5.8 \mathrm{GHz}$ [24], and a prototype of a NFF reflectarray operating at $140 \mathrm{GHz}$ has been realized and characterized in [25]. Moreover, a $5.8 \mathrm{GHz}$ NFF reflectarray with multifocus characteristics has been designed and tested in [26]. It has been shown that a single reflectarray can be used to implement a single-feed multi-focus antenna, or a multi-feed multi-focus antenna. Another multi-focus antenna has been presented by Chou in [27], where the outputs of a Rotman lens feed eight linear NFF patch arrays at $2.45 \mathrm{GHz}$.

A number of recent papers deal with reconfigurable NFF antennas. Li and his coauthors [28] designed a microstrip NFF linearly-polarized array with a frequency-tunable focal distance, by exploiting a series-feeding along both the $\mathrm{E}$ and $\mathrm{H}$ planes. The proposed antenna can move the field peak along the array broadside direction, for a distance to the array surface going from 78 to $249 \mathrm{~mm}$, as frequency increases from 9.25 to $10.5 \mathrm{GHz}$. The proposed configuration does not require any phase shifter and performances as other frequency-dependent NFF antennas, as for example leaky-wave antennas. In a previous paper [29], the authors showed a microstrip NFF array antenna with a steerable focus on the focal plane. The focus scanning ability along both orthogonal directions of the focal plane are achieved by phase control and frequency shift, respectively. A further layout that combines the frequency scanning in one plane with a phase shift in the orthogonal plane is in [30], where a Ka-band single-layer NFF SIW (Surface Integrated Waveguide) array antenna is realized. There, a set of parallel NFF leaky-wave SIW linear slot antennas are fed through a focused Rotman lens. In this case, the NFF beam can be scanned in the H-plane by changing the frequency and in the other plane by changing the input port of the Rotman lens. Finally, Yurduseven [31] showed a detailed numerical analysis of the performance of a holographic metasurface using PIN 
diodes for implementing a NFF antenna with reconfigurable foci.

\section{Discussion and Conclusions}

Focusing and shaping of the electromagnetic field in a region close to antenna can be beneficial for several shortrange wireless applications, as demonstrated from the incessant research activity on design criteria and technologies for the implementation of near-field focused antenna. The basic concept is to sum in phase (constructive interference) all the field contributions from a continuous or discrete aperture at an assigned focal spot in front of the antenna, from which the well-known conjugate-phase method is easily derived. Many near-field focused antennas are based on that systematic design approach, regardless of the adopted antenna technology (microstrip arrays, transmitarrays and reflectarrays, dielectric resonator antennas, leaky-wave antennas, Fresnel plate lenses, etc). Nevertheless, advanced wireless systems may require for a finer shaping of the near field, and then pretty much novel synthesis techniques have been applied to control the depth-of-focus, side-lobe level, size and shape of the focal region in the transverse plane, multiple-focus location and their relative amplitude, etc. Also, more and more design procedures will be required to face with future challenging near-field requirements. Nevertheless, the phase profile coming from the simple conjugate-phase approach can still serve as an effective starting solution for any optimization approach, regardless of the antenna technology and frequency specifications. The powerful of the conjugatephase approach is also given by the equivalence of the farfield pattern and the pattern at the focal plane, which allows for an easy rearrangement of the several far-field pattern synthesis techniques developed during the last decades.

Most of the proposed solutions optimize the near field when neglecting some important electromagnetic phenomena: array coupling, interaction with obstacles close to the focusing antenna, effects of the target device/scatterer at the focal region, variations with frequency of the focusing performance, non-homogeneity of the material surrounding the antenna. Sometimes, some of the above effects are evaluated a posteriori, namely using the solution optimized under ideal conditions. On the other hand, the application of NFF antennas in real scenarios cannot neglect above phenomena and, in some critical designs, at least some of them should be accounted since the first steps of the design process.

Finally, it is worth noting that in some practical scenarios the assigned focal region of interest could be closer to the antenna surface and then out of the radiative near-field region. In such situations, the assessment of the antenna radiated field cannot be done through analytical techniques, and full-wave numerical simulations are typically mandatory. Also, the variations of the input impedance of the focused antenna when the target is present into the assigned focal region are not negligible, and the antenna design process cannot consider just the near-field shaping, since return loss and bandwidth performance, as well as coupling phenomena, must be also considered concurrently. In this context, it is desirable the development of advanced design procedures and tools that may account, simultaneously and hopefully in a simple way, for most of the above mentioned issues. Other hot topics in the context of NFF antennas are about antenna layouts for dynamic focusing, or synthesis techniques for the concurrent optimization of the antenna far-field pattern and shaping of the near field in one or more assigned regions close to the antenna surface.

\section{Acknowledgements}

This work has been supported by the Mobility Grants of the University of Oviedo financially supported by Banco de Santander and by the FPU grant of the Spanish Ministerio de Educación, Cultura y Deporte with reference FPU15/06431.

\section{References}

1. P. Nepa, A. Buffi, "Near-Field Focused Microwave Antennas," IEEE Antennas and Propagation Magazine, 59, no. 3, pp. 42-53, June 2017.

2. P. Nepa, A. Buffi, A. Michel, G. Manara, "Technologies for Near-Field Focused Microwave Antennas," International Journal of Antennas and Propagation, Vol. 2017, 17 pages, doi: 10.1155/2017/7694281, 2017.

3. A. Buffi, P. Nepa, G. Manara, "Design Criteria for NearField-Focused Planar Arrays," IEEE Antennas and Propagation Magazine, 54, no. 1, pp. 40-50, Febr. 2012.

4. L. Shan, W. Geyi, "Optimal design of focused antenna arrays," IEEE Trans. Antennas Propag., 62, n.11, pp. 5565-5571, Nov. 2014.

5. H. C. Sun, W. Geyi, "Optimum design of wireless power transmission systems in unknown electromagnetic environments," IEEE Access, 5, pp. 20198-20206, 2017.

6. H. P. Tong, W. Geyi, “Optimal design of smart antenna systems for handheld devices," IET Microwaves, Antennas and Propagation, 10, n. 6, pp. 617-623, Apr. 2016.

7. X. D. Yang, W. Geyi, H. Sun, "Optimum design of wireless power transmission system using microstrip patch antenna arrays," IEEE Antennas and Wireless Propagation Letters, 16, pp. 1824-1827, Mar. 2016.

8. Y. H. Jiang, W. Geyi, L. S. Yang, H. C. Sun, "Circularlypolarized focused microstrip antenna arrays," IEEE Antennas Wireless Propagation Lett., 15, pp. 52-55, 2016.

9. Xiao Cai, Wen Geyi, "An optimization method for the synthesis of flat-top radiation patterns in the near-field and far-field regions," to appear on IEEE Trans. on Antennas and Propagation, 2018. 
10. R. G. Ayestarán, M. R. Pino, P. Nepa, "Synthesis of near field focused arrays including far-field constraints," 2017 International Applied Computational Electromagnetics Society Symposium (ACES), Florence, Italy, pp. 1-2, 2017.

11. Z. X. Huang and Y. J. Cheng, "Near-Field Pattern Synthesis for Sparse Focusing Arrays Based on Bayesian Compressive Sensing and Convex Optimization," IEEE Transactions on Antennas and Propagation, 66, n. 10, pp. 5249-5257, October 2018.

12. R. G. Ayestarán, "Fast Near-Field Multifocusing of Antenna Arrays Including Element Coupling Using Neural Networks," IEEE Antennas and Wireless Propagation Letters, 17, n. 7, pp. 1233-1237, 2018.

13. G. G. Bellizzi, D. A. M. Iero, L. Crocco, T. Isernia, "Three-Dimensional Field Intensity Shaping: The Scalar Case," IEEE Antennas and Wireless Propagation Letters, 17, n. 3, pp. 360-363, 2018.

14. D. A. M. Iero, L. Crocco, T. Isernia, "Constrained power focusing of vector fields: An innovative globally optimal strategy," J. Electromagnetic Wave, 29, n. 13, pp. 1708-1719, 2015.

15. G. G. Bellizzi, M. T. Bevaqua, L. Crocco, T. Isernia, "3-D Field Intensity Shaping via Optimized Multi-Target Time Reversal," IEEE Transactions on Antennas and Propagation, 66, n. 8, pp. 4380-4385, August 2018.

16. I. Iliopoulos, B. Fuchs, R. Sauleau, P. Pouliguen, P. Potier, M. Ettorre, "On the use of convex optimization for electromagnetic near-field shaping, " 2017 11th European Conference on Antennas and Propagation (EUCAP), pp. 1013-1016, 2017.

17. I. Iliopoulos, B. Fuchs, R. Sauleau, P. Pouliguen, P. Potier , M. Ettorre, "Scalar near-field focusing in lossy media," 2017 International Conference on Electromagnetics in Advanced Applications (ICEAA), pp. 718-721, Verona, Italy, 2017.

18. G. Xu R. Zeng, S. Chen, M. Shi, C. Yu, "A novel synthesis method for millimeter-wave antenna with contoured-beam at near-field region," 2017 47th European Microwave Conference (EuMC), pp. 264-267, 2017.

19. W. Wang, H. Gao, Y. Wu, Y. Liu, "Impact of amplitude weights on power focusing for near - field - focused planar arrays," International Journal of RF and Microwave Computer-Aided Engineering, pp. 1-13, February 2018.

20. I. Iliopoulos, M. Casaletti, R. Sauleau, P. Pouliguen, P. Potier, M. Ettorre, "3-D shaping of a focused aperture in the near field," IEEE Transactions on Antennas and Propagation, vol. 64, no. 12, pp. 5262-5271, Dec. 2016.
21. S. H. Zainud-Deen, W. M. Hassan and H. A. Malhat, "Near-Field Focused Folded Transmitarray Antenna for Medical Applications," Wireless Personal Comm., Issue 3, pp.4885-4894, May 2017.

22. V. R. Gowda, M. F. Imani, T. Sleasman, O. Yurduseven, D. R. Smith, "Focusing Microwaves in the Fresnel Zone With a Cavity-Backed Holographic Metasurface," IEEE Access, 6, pp. 12815 - 12824, 2018.

23. H.-T. Chou, J.-W. Liu, C.-Y. Liu, P. Nepa, "Focusing characteristics of near-field radiations from multi-panels of phased array of antennas in circularly cylindrical arrangement," 2017 International Symposium on Antennas and Propagation (ISAP), pp. 1-2, Phuket, Thailand, 2017.

24. G. S. Lipworth, J. A. Hagerty, D. Arnitz, Y. A. Urzhumov, D. R. Nash, R. J. Hannigan, C. T. Tegreene, M. S. Reynolds, "A Large Planar Holographic Reflectarray for Fresnel-Zone Microwave Wireless Power Transfer at 5.8 GHz," 2018 International Microwave Symposium, pp. 964967, 2018.

25. F. Zhao and Y. J. Cheng, "Near-field focused reflectarray antenna at $140 \mathrm{GHz}$," 2018 IEEE MTT-S International Wireless Symposium (IWS), pp. 1-4, 2018.

26. S. Yu, H. Liu, L. Li, "Design of Near-Field Focused Metasurface for High Efficient Wireless Power Transfer with Multi-Focus Characteristics," to appear on IEEE Transactions on Industrial Electronics, 2018.

27. H.-T. Chou and Z.-C. Tsai, "Near-Field Focus Radiation of Multi-Beam Phased Array of Antennas Realized by Using Modified Rotman Lens Beamformer, to appear on IEEE Transactions on Antennas and Propagation, 2018.

28. P.-F. Li, S.-W. Qu, S. Yang, Y.A. Liu, Q. Xue, "NearField Focused Array Antenna With Frequency-Tunable Focal Distance," IEEE Transactions on Antennas and Propagation, 66, no. 7, pp. 3401 - 3410, July 2018.

29. P.-F. Li; S.-W. Qu, S. Yang, Z.-P. Nie, "Microstrip Array Antenna With 2-D Steerable Focus in Near-Field Region," IEEE Transactions on Antennas and Propagation, 65, n. 9, pp. 4607-4617, 2017.

30. Y. F. Wu, Y. J. Cheng and Z. X. Huang, "Ka-Band Near-Field-Focused 2-D Steering Antenna Array With a Focused Rotman Lens," IEEE Transactions on Antennas and Propagation, 66, n. 10, pp. 5204-5213, October 2018.

31. O. Yurduseven, D. L. Marks, J. N. Gollub, D. R. Smith, "Design and Analysis of a Reconfigurable Holographic Metasurface Aperture for Dynamic Focusing in the Fresnel Zone," IEEE Access, 5, pp. 15055 - 15065, 2017. 